Aus der chirurgischen Universitätsklinık der Charité zu Berlin. (Direktor: Geh. Med.-Rat Prof. Dr. Hildebrand.)

\title{
Zur künstlichen Epithelisierung der Witzelfistel nach Narath.
}

\author{
Von Dr. Gustav Hauck, Assistent der Klinik.
}

Die von Narath in Bd. I54, S. 4I 8 dieser Zeitschrift vorgeschlagene Auskleidung des Wiłzelschen Schrägkanals mit Thierschschen Läppchen wurde hier zweimal ausgeführt, bei einer Jejunum- und einer Magenfistel. In beiden Fällen wurde nach Eröffnung der Bauchhöhle von der Streckseite des Oberschenkels ein etwa fingerlanges und halb so breites Thierschsches Läppchen entnommen und um das untere Ende des Gummischlauches so herumgelegt, daß die Wundfläche nach außen zu liegen kam, die untere Öffnung des Schlauches aber frei blieb. Das so armierte Gummirohr wurde dann in den aus Darm- bzw. Magenwand und Bauchmuskulatur gebildeten Schrägkanal in der bekannten Weise eingenäht. Der Träger der Jejunumfistel starb nach 50 Stunden. Im Sektionspräparat 1) war das Thierschsche Läppchen etwas nach innen verschoben und kleidete den Kanal nur noch bis zur Hälfte aus, jedoch lag es bereits auf der Darmserosa so fest, daß es sich weder beim Spülen noch bei der Anfertigung der Gefrierschnitte nach vorausgegangener Formalinhärtung von dieser sichtbar ablöste. Mit der Pinzette hingegen ließ es sich von seinen Endzipfeln aus ohne weiteres abtragen. Mikroskopisch war aber dann auch die Darmserosa mitabgehoben, die am Thierschschen Läppchen festhing und die Muscularis dadurch bloßlegte; genau so hatte sich das Läppchen an einigen anderen kleinen Stellen vermutlich durch stumpfe Gewalteinwirkungen, sei es durch das Fixieren oder Schneiden des Präparates nur im Zusammenhang

I) Für die Überlassung der Präparate bin ich Herrn Geheimrat Lu ba $\mathrm{rsch} z \mathrm{u}$ großem Danke verpflichtet. 
mit dè Serosa gelockert. Im ganzen bestand eine unverkenubar innige Verbindung zwischen dem aufgepflanzten Thierschschen Läppchen und der Iarmwand; hie und da lagen noch kleine flache Blutkoagula zwischen beiden eingestreut, die die Grenze noch deutlich erkennen ließen. Die Zellkerne des der Tunica propria entstammenden bindegewebigen Anteils des Läppchens und des von ihm getragenen Plattenepithels waren gleichmäligr und kräftig gefärbt, nur da, wo das Läppchen durch Überlage. rung, Uberhängen und Faltenbildung nicht mit der Serosa in Ver. bindung hatte treten konnen, herrschte ein allgemeiner Kernschwund und eine hyaline Entartung der Bindegewebsfasern vor. Ein schmales ringförmiges Geschwür stellt an der inneren Mun. dung des Kanals den Ubergang zwischen Läppchen und Schleim. haut dar. Die Magenfistel wurde in gleicher Weise wie die Jeju. numfistel angelegt. Nach einer anfänglich starken Sekretion aus dem Wundkanal wurde nach I + Tagen der erste Schlauchwechsel vorgenommen. Der Witzelkanal lieb, soweit er dem Auge zugäng. lich war, eine gleiclimäßige Epithelauskleidung erkennen, nur an seiner äußeren Mündung befand sich seitlich noch eine linsengroße Granulation, die sich nach einiger Zeit ebenfalls mit Epi. thel überzog. Die Sekretion war von nun ab ganz minimal; die äußere Fistelöffnung und ihre Umgebung blieben unter dem Ver. bande dauernd trocken, Mazeration der Haut durch ausgetretenen Magensaft trat nicht mehr auf. Die Wiedereinführung des Schlauches war nur einmal nach einer langeren Pause von 12 Stunden etwas schwieriger. Nach nahezu 3 Monaten starb der Patient (6. IV. bis 25.VI. 1921). Im Sektionspräparat ist auch die innere Mündung das Kanals mit einer dicken grauweißen Plattenepithelschicht ausgekleidet, die sich noch in 1,2 pfennigstückgroßer Ausdehnung in die Umgebung ausbreitet, um dann direkt in die mit ihr fest verwachsene Magenschleimhaut uberzugehen. Der der Länge nach aufgeschnittene Kanal mißt $3^{1}, 2 \mathrm{~cm}$ im Längs-, I cm im Querdurchmesser und verläuft um nahezu $3 \mathrm{~cm}$ schräg nach oben und I cm medial. Er ist in ganzer Länge versehen mit einem dicken. starren Epithelrohr, das die Lichtung klaffend hält; ungefähr in der Mitte des Kanals findet sich an der medialen Seite ein pfennig. stückgroßer flacher geschwüriger Defekt der Epithelwand und ihm gegenüber ein vermutlich durch die Naht an der Crenze zwischen 
Magen- und Bauchwand entstandene ziptelig ausgczngene Nische, in die aber das Epithel ohne Unterbrechung übergeht. Die Verwachsung des Epithels auf seiner Serosa- und Muskelunterlage ist überall so fest, dals es sich durch keinerlei stumpfe ricwalt abheben läßt. Mikroskopisch ist das uberpflanzte Lappchen in der Epithel- und Bindegewebsschicht durch Zellermehrung etwa auf das Doppelte rerdickt; im bindegewebigen Anteil finden sich reichliche, prall mit Blut gefullte Blutgefabe und einige Rund. zelleninfiltrate. Lie Verwachsung mit der Serosa bezichungsweise Bauchwandmuskulatur ist so vollkommen, daß die Grenze nur noch durclı dic Verschiedenheit der Gewebe zu erkennen ist. Am Übergang zur Magenschleimhaut ist das Plattenepithel und ebenso das Bindegewebe des Läppchens wallartig verdickt und geht so direkt in das Drusenepithel der Magenschleimhaut und die Sub. mucosa über. In die äußere Haut setzt sich die Epithelauskleidung ohne Unterbrechung fort, wobei es sich langsam zur gewöhnlichen Epitheldicke verjungt. Das erwahnte Geschwür in der Mitte des Kanals ist lediglich ein Epitheldefekt mit herdformigen, chronisch entzindlichen Infiltrationen des bindegewebigen (irundes.

Das Thierschsche Läppchen zeigte also in den beiden Fallen die ausgesprochene Tendenz rasch und fest mit der Wand des Witzelschen Schrägkanals zu verwachsen. Die Epithel- und Bindegewebsvermehrung ist wohl aut die dauernde, durch den Druck des Schlauches heriorgerufene Reizung zururkzufuhren. Inwicweit nach der Entfernung des Schlauches eine Vercngerung des Kanals eintritt, konnte in diesem Falle nicht nachgepruft werden, da der Patient, wie man das häufig trufft, beım Schlauchwechsel ängstlich um die sofortige Wiedereinführung bat. Daßs sie emmal nach einer 12 stündigen Pause etwas schwieriger war, mags wohl an cler Quellung der von Druck befreiten Cewebe liegen. Eine Verengerung und ein Verschlul. des epithelisierten Kanals tritt, wenn überhaupt, sicherlich viel langsamer ein, als beim nichtepithelsierten, da schon die Epithelauskleidung mit ihrer bindegewebigen Unterlage wic eine stutzende Einlage die Wand des Kanals von vornherein am Kollabieren verhindert, ganz abgesehen von dem völligen Fehlen einer zur Schrumpfung neigenden Wundflache.

Dem Augenschein nach bot die Magenfistel bes der Sektion ncch ungefähr dasselbe Bild in bezug auf Lange und Richtung 
wie direkt nach der 3 Monate vorher erfolgten Operation. Obwohl der bei der Geradestellung der Witzelfistel von $\mathrm{Nar}$ at h als der Hauptschuldige angesprochene Gummischlauch fast ununterbrochen 3 Monate hindurch liegen geblieben war, war die Verlaufsrichtung des Kanals nicht merklich verändert bei der Sektion. Ebenso war auch keine Verkürzung eingetreten, die sonst mit der Geradestellung gleichen Schritt halt. Scheinbar wird auch dieser ProzeB durch die Epithelisierung zum mindesten verschleppt.

Die beiden Momente, die Narath zu dem Vorschlag der Epithelisierung einer Dauer-Witzelfistel bewogen haben, erstens die Verengerung und zweitens die Verkürzung und Geradestellung des Kanals sind also hier nicht eingetreten. Durch die Epithelisierung wurde aber weiterhin noch etwas erreicht, was besonders vom $\mathrm{Pa}$ tienten sehr angenehm empfunden wird, namlich das Versiegen der Sekretion. Die nicht epithelisierten Fistelgänge sind mit Granulationen ausgekleidet, die sich neben dem Schlauche aus der Fistel herausdrangen und eine mehr oder minder starke eitrige Sekretion unterhalten. In den weichen Granulationen schließt der Schlauch nicht so gut ab wie in dem festen Plattenepithelrohr, der Austritt von Magensaft und die Mazeration der Haut sind die Folgen, die den Fistelträger in eine qualvolle Lage versetzen. Es war zwar bei der Magenfistel die ersten 10 Tage nach der Operation eine starke Sekretion eingetreten, aber nachdem anscheinend das Läppchen völlig angewachsen und die überhängenden Teile desselben ausgestoßen waren, hat auch die Sekretion nachgelassen und war schließlich völlig ausgeblieben. Der Schlauch lag absolut dicht. Die Fistel glich mehr einer natürlichen Öffnung; das Fehlen jeglicher Granulation und Eiterung nahm dem Patienten das subjektive Gefühl einer Wunde. Der sonst notwendige häufige Verbandwechsel fiel gänzlich fort und es war die Behandlung dadurch wesentlich vereinfacht.

Es bedeutet also die Epithelisierung der Witzelfistel sowohl für den Arzt als auch besonders für den Patienten eine große Erleichterung und man sollte deshalb die kleine Mühe, die die Auskleidung des Kanals mit einem Thierschschen Läppchen bereitet, nicht scheuen. Selbstverstandlich gilt dies nur für die Anlegung von Dauerfisteln. Ich habe mit einem entsprechend großen 
Läppchen das untere Schlauchende umhüllt und den uberlagernden Rand nicht abgetragen, sundern einfach weiter ringsum angelegt. Der Zug der elastischen Iasern un bindegewebigen Anteil des Läppchens und die Adhäsion machen eme besondere Fixierung uberflüssig. Jer Schlauch mul3 beim Innàhen sehr ruhig gehalten werden und rlie crsten Nähte mussen besonders fest sitzen Weiterhin ist es wo Vorleil, wie Narath empfohlen hat, den „Krater” ringsherum an die iulbere Haut einzunähen; es entsteht so eine Kanalwand aus einem Stuck und die Serosa bietet dem Thierschschen Läppchrol eine uncrgleichlin h ganstigt: Inhat tungsfläche.

Das Resultat dieser beaden Fäll r rmutiol \& weiteren Verauhen. 